\title{
CORRELAÇÃO DE DADOS GEOFÍSICOS EM PESQUISA MINERAL DE OCORRÊNCIA DE COBRE EM CAÇAPAVA DO SUL (RS)
}

\author{
GEOPHYSICAL DATA CORRELATION IN MINERAL EXPLORATION OF COPPER \\ OCURRENCE IN CAÇAPAVA DO SUL (RS)
}

\author{
Henrique Garcia PEREIRA ${ }^{1}$, César Augusto MOREIRA², Pedro Lemos CAMARERO ${ }^{3}$ \\ ${ }^{1,3}$ Universidade Estadual Paulista - UNESP. Programa de Pós-graduação em Geociências e Meio Ambiente. Rio Claro - SP. Emails: \\ ique_garcia@hotmail.com, camarero@folha.com.br \\ ${ }^{2}$ Universidade Estadual Paulista -UNESP. Departamento de Geologia Aplicada. Rio Claro - SP. Email: moreirac@rc.unesp.br
}

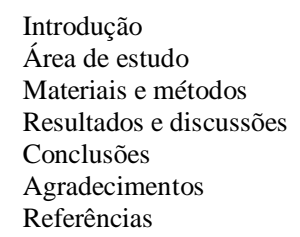

RESUMO - O cobre é um metal extremamente utilizado em diversos setores da produção industrial e da construção civil. O crescimento social e tecnológico vivido pela sociedade contemporânea tem levado diversas reservas conhecidas desse bem mineral metálico ao exaurimento. A busca por novas jazidas ou a reavaliação de ocorrências já conhecidas se torna cada vez mais importantes. Nesse sentido, a geofísica pode ser utilizada como ferramenta de investigação para melhores estimativas do potencial mineral de subsuperfície. Este trabalho descreve o uso de ensaios de caminhamentos elétrico e eletromagnético para a avaliação de uma provável continuidade lateral sudoeste das mineralizações da ocorrência cuprífera Capão Grande. A investigação de subsuperfície foi realizada através de quatro perfis dispostos perpendicularmente à ocorrência mineral. Os modelos de inversão provenientes do caminhamento elétrico permitiram a identificação de áreas centrais de baixa resistividade para os perfis 2, 3 e 4, possivelmente associadas a mineralização em subsuperfície, sugerindo sua continuidade para sudoeste da ocorrência. As seções de condutividade aparente possibilitaram a estimativa da presença de sulfetos em subsuperfície, quando houve correlações de alta condutividade com as áreas prévias de baixa resistividade associadas a mineralização.

Palavras-chave: cobre; geofísica; continuidade lateral; baixa resistividade; alta condutividade.

\begin{abstract}
Copper is a metal extremely used in various sectors of the industrial production and civil construction. Social and technological growth experienced by contemporary society has led several known reserves that right to metallic mineral soil. The search for new deposits or reevaluation of previously known occurrences becomes increasingly important. In this sense, the Geophysics can be used as a research tool for better estimates of subsurface mineral potential. This paper describes the use of electric and electromagnetic walking tests for the evaluation of a probable continuity of mineralization in the southwest side mineralized copper occurrence Capão Grande. The subsurface investigation was performed through four profiles arranged perpendicularly to the mineral occurrence. Inversion models from walking electric allowed the identification of central areas of low resistivity for profiles 2 , 3 and 4, possibly associated with subsurface mineralization, suggesting its continuity to southwest of the occurrence. The apparent conductivity sections allowed the estimation of the presence of sulfides in subsurface, when there were high correlations with previous areas of conductivity low resistivity associated with mineralization.
\end{abstract}

Keywords: copper; geophysics; lateral continuity; low resistivity; high conductivity.

\section{INTRODUÇÃO}

A importância de estudos envolvendo a pesquisa mineral de cobre advém de sua grande aplicabilidade em diversos setores da produção, em virtude de suas características técnicas, como alta condutividade elétrica e térmica, excelente resistência à corrosão, alta plasticidade, facilidade de manufatura e de liga com outros metais, ótimas características abrasivas e de soldagem, além de excelentes condições para a deposição eletrolítica (Chatterjee, 2007).

Uma área com amplo potencial para a produção desse metal básico é a do município de Caçapava do Sul, localizado na porção centro-sul do estado do Rio Grande do Sul. Nessa região são reconhecidas diversas jazidas e ocorrências de cobre associadas preferencialmente às rochas vulcanossedimentares e metavulcanoclásticas (Porcher et al., 1995).

Muitas dessas jazidas foram amplamente estudas, como a de Camaquã, que foi explorada desde o início do século XIX até o esgotamento da mineralização na década de 1990 (Ronchi et al., 2000). Por outro lado, ocorrências como Capão Grande carecem de estudos quanto ao seu potencial mineral em subsuperfície.

O potencial mineral de subsuperfície pode ser avaliado diretamente através da abertura de trincheiras e por sondagens rotativas, ou indiretamente pela geofísica (Moon et al., 2006). Os métodos geofísicos constituem uma ferramenta de alta aplicabilidade na pesquisa mineral, principalmente nos casos de sulfetos 
metálicos de interesse econômico (maciços e/ou disseminados), pois permite detectar contrastes de propriedades físicas entre um alvo potencialmente mineralizado e a rocha encaixante (Kearey, 2002).

Os métodos geofísicos tradicionalmente empregados na pesquisa mineral de metais básicos são a eletrorresistividade e a polarização induzida, os eletromagnéticos nos domínios do tempo e da frequência, além da magnetometria (Pereira, 2003). O uso combinado destes métodos na pesquisa de sulfetos apresenta resultados bastante satisfatórios, conforme descrito nos trabalhos de Garrido (1978), Sá (1981), Allis (1990), Irvine \& Smith (1990), Write et al. (2001), Moreira \& Ilha (2011), Moreira et al. (2012).

O presente trabalho apresenta e discute a aplicação conjunta de dados geofísicos da eletrorresistividade e do eletromagnético no domínio da frequência desenvolvida para verificar uma provável continuidade lateral sudoeste das mineralizações da ocorrência cuprífera Capão Grande.

\section{ÁREA DE ESTUDO}

A área de estudo está localizada a noroeste da sede do município de Caçapava do Sul (RS), que fica a $259 \mathrm{~km}$ da capital Porto Alegre pela BR 290. O acesso à área a partir do município é realizado pela BR 392 por $17 \mathrm{~km}$ até o entroncamento com a BR 290, onde essa é acessada para oeste por $15 \mathrm{~km}$ até a Vila do Cerrito do Ouro. A partir dessa localidade tomase a estrada municipal Caçapava/Lavras para o sul por $9 \mathrm{~km}$ até a área de estudo (Figura 1).

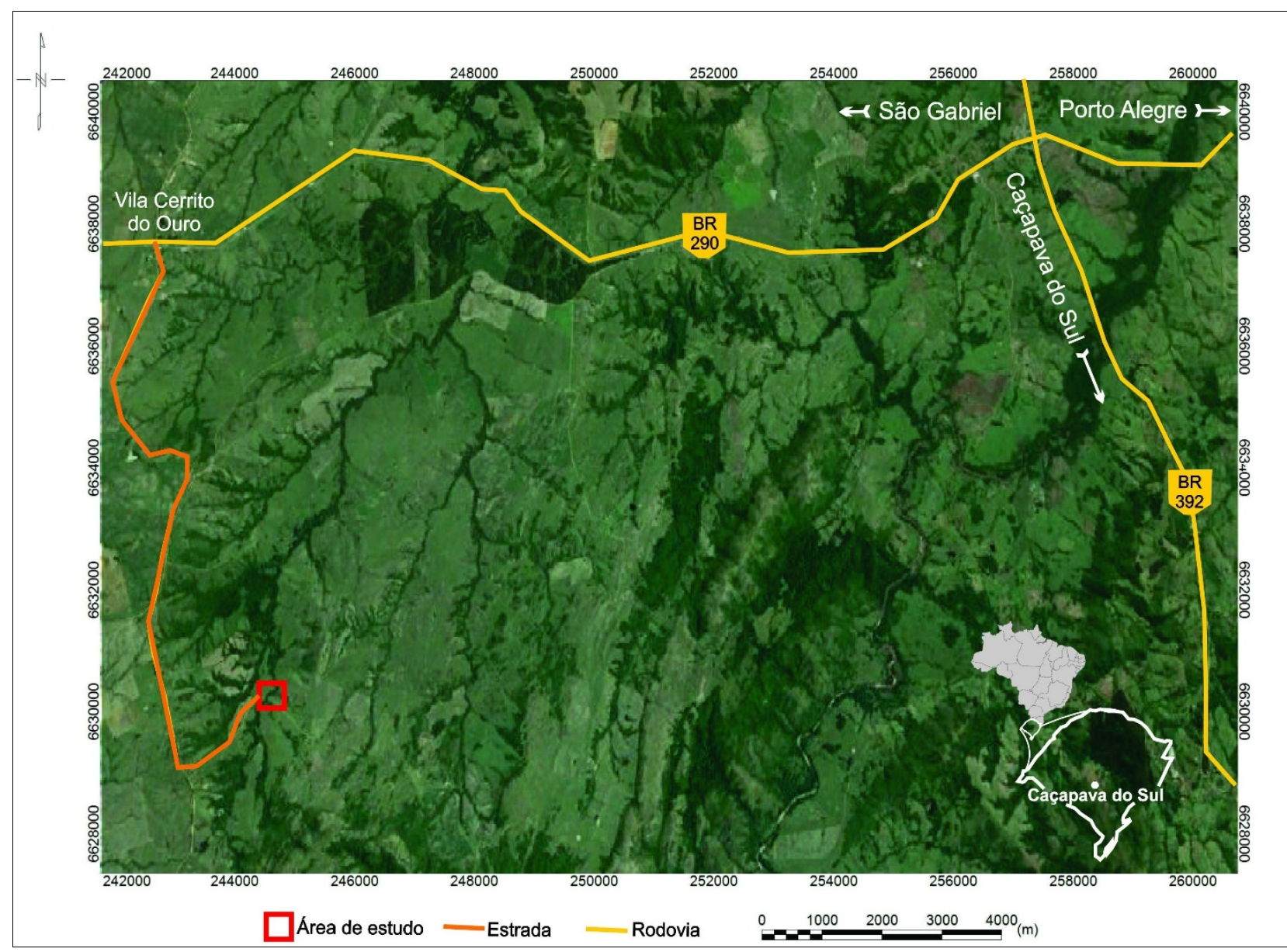

Figura 1 - Localização da área de estudo.

A ocorrência cuprífera Capão Grande foi descrita pelo Departamento Nacional de Produção Mineral (DNPM) em 1969 como sendo do tipo disseminada (Bocchi, 1970). Essa ocorrência está inserida em rochas vulcanossedimentares da Formação Passo da
Promessa, pertencentes ao grupo Maricá da Bacia do Camaquã. O embasamento dessa formação é constituído por rochas metavulcanoclásticas do Complexo Metamórfico Vacacaí, unidade integrante do escudo cristalino Sul-RioGrandense (Figura 2). 


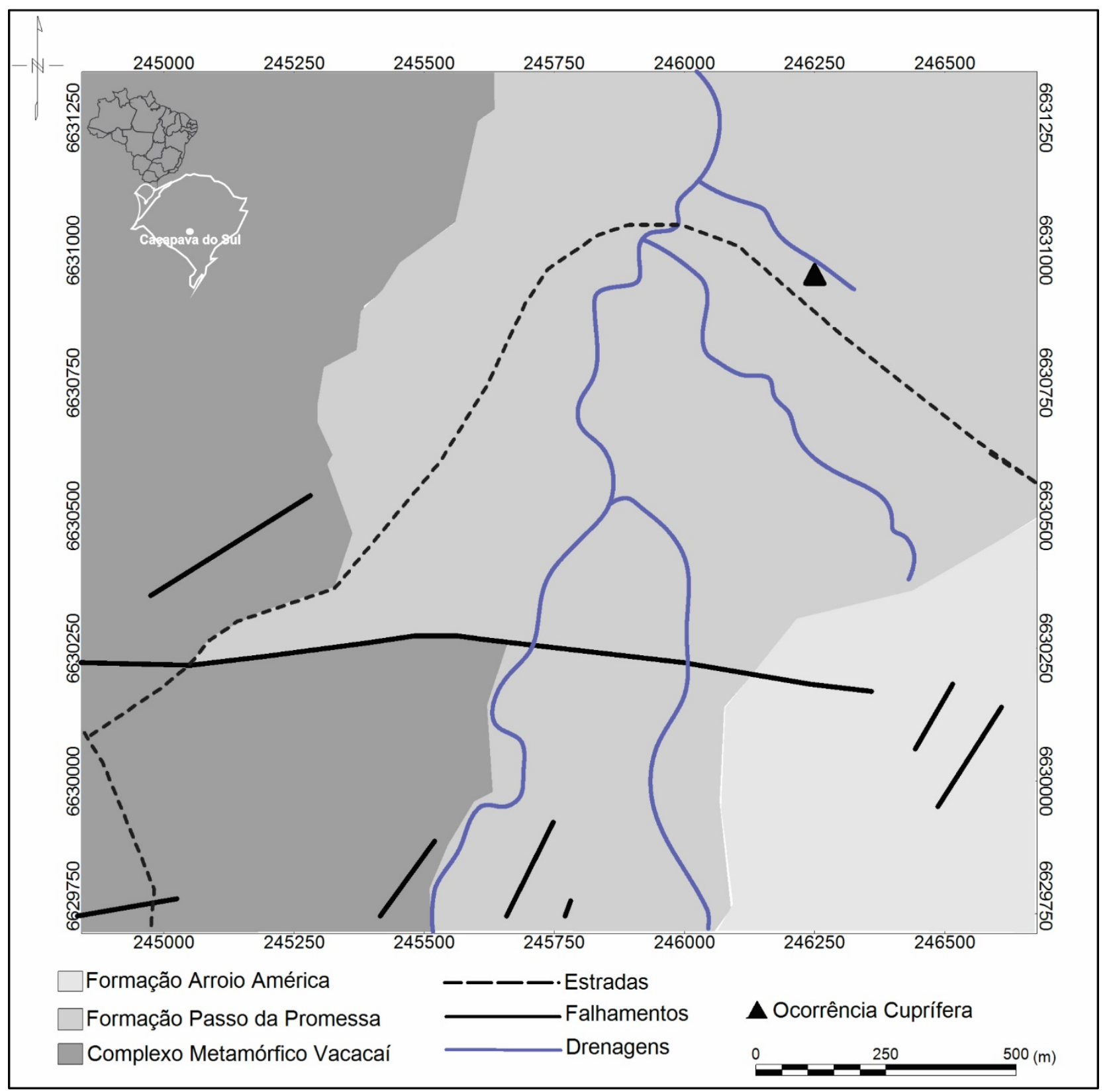

Figura 2 - Contexto geológico e ocorrência abordada no estudo (Adaptado de Porcher et al., 1995).

De acordo com Porcher et al. (1995), a unidade metavulcanoclástica do Complexo Metamórfico Vacacaí é constituída predominantemente de rochas vulcanoclásticas, às quais se associam a rochas epiclásticas e químicas, que foram afetadas pelo contato com rochas supracrustais, cujo metamorfismo gerou condições de fácies xisto verde inferior para médio a superior, zona da clorita.

Vestígios de fácies metamórficas mais elevadas, geradas por metamorfismos de contato, também são registradas, como hidrotermalismo, silicificação e carbonatização, responsáveis pelas fácies hornblenda hornfels, impostos princ.ipalmente por intrusões graníticas brasilianas póstectônicas mais jovens (Porcher et al., 1995).
A Formação Passo da Promessa é constituída por arenitos róseos a avermelhados, cinzaavermelhados ou castanho-avermelhados. Apresentam com frequência níveis conglomeráticos, onde os seixos são predominantemente de rochas graníticas e metamórficas. Seixos de quartzo são raros e estão imersos e isolados na matriz arcosiana, e normalmente dispostos em níveis preferenciais segundo o acamamento da rocha (Bocchi, 1970; CPRM, 2007).

O afloramento Capão Grande apresenta 19 $\mathrm{m}$ de comprimento por $10 \mathrm{~m}$ de espessura, e se dispõe na direção NW (Figura 3a). É constituído por arenitos arcosianos conglomeráticos, que apresentam impregnações 
de malaquita $\left(\mathrm{Cu}_{2} \mathrm{CO}_{3}(\mathrm{OH})\right)$ e azurita $\left(\mathrm{Cu}_{3}\left(\mathrm{CO}_{3}\right)_{2}(\mathrm{OH})_{2}\right)$ ao longo de fraturas (Figura 3b) e também em porções mais porosas da rocha (Figura 3c).

Estudos sobre a gênese da mineralização da ocorrência cuprífera Capão Grande não foram encontrados na literatura. A mineralização de cobre mais próxima que já foi alvo de desse tipo de estudo é a da Mina do Camaquã. A evolução das hipóteses propostas para a origem das mineralizações dessa mina foi decorrente da aquisição de novos dados vinculados à lavra do minério sulfetado.

A presença de filões espessos no início das operações fez com que o modelo hidrotermalmagmático fosse admitido por Teixeira (1937), Leinz \& Almeida (1941) e Costa Filho (1944). Ainda segundo tais autores, as mineralizações cupríferas “clássicas” encontradas em toda Bacia do Camaquã mostram distribuição regional marcadamente linear, segundo falhamentos na direção N20E.

Conforme o avanço da lavra, o modelo sedimentar singenético a diagnético passou a ser mais aceito devido às concentrações expressivas de sulfetos disseminados e estratiformes. Perto da exaustão das reservas econômicas, o minério sulfetado diminuiu, os teores de ouro aumentaram e o modelo epitermal passou a ser considerado como o mais adequado (Laux \& Lindenmayer, 2000).

A partir das características estratigráficas, geoquímicas e isotópicas, Laux et al. (2005) propuseram a origem hidrotermal-epitermal ou, alternativamente, hidrotermal-magmática distal para as Minas do Camaquã. Até o advento de estudos mais profundos, este parece ser o modelo metalogenético mais consistente para esta região.
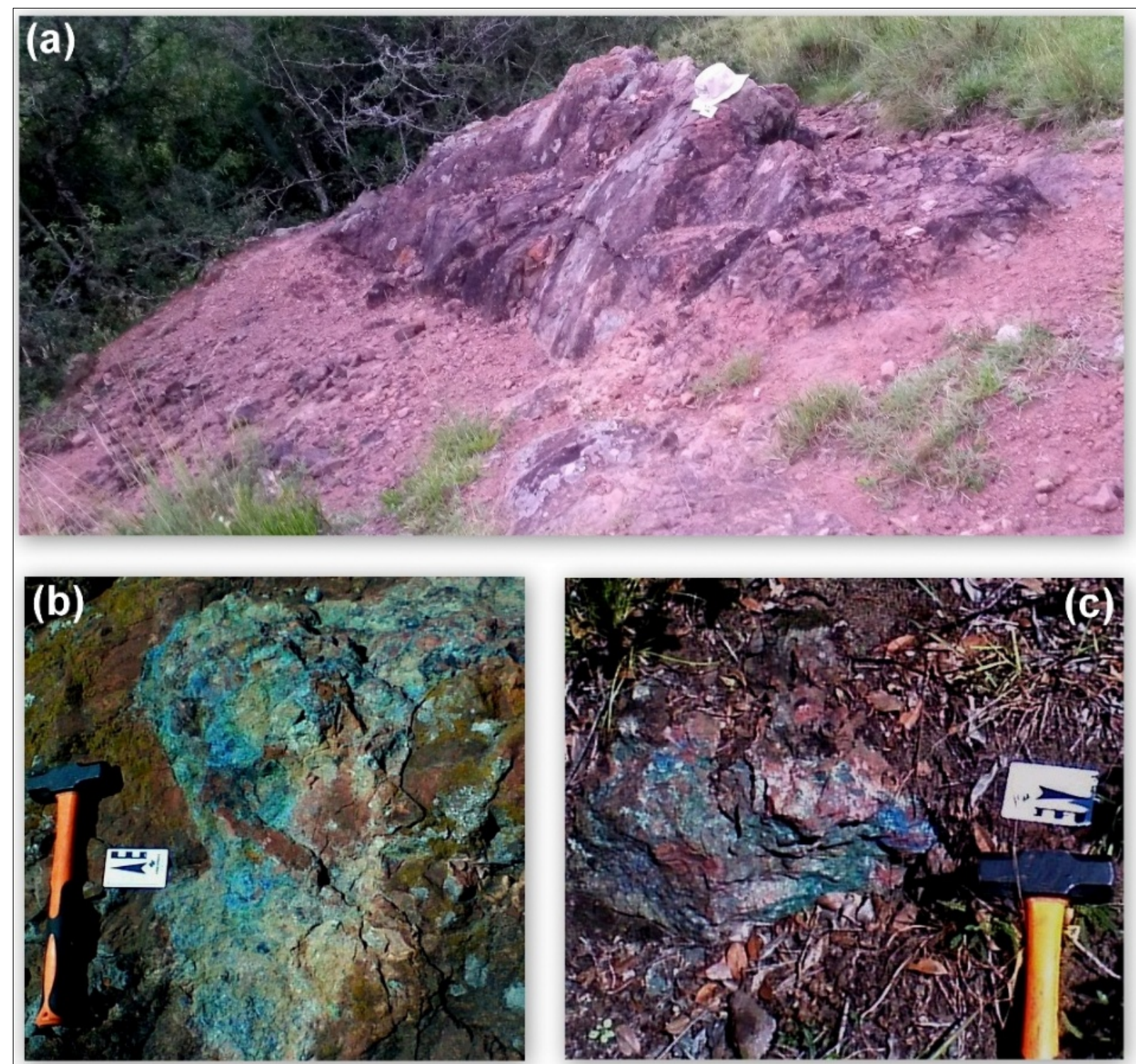

Figura 3 - Características do afloramento Capão Grande: a) disposição NW; b) carbonatos em fraturas; c) carbonatos em zonas de maior porosidade. 


\section{MATERIAIS E MÉTODOS}

Os equipamentos utilizados nesse estudo foram o resistivímetro Syscal Pro da Iris Instruments e o condutivímetro de terreno EM34 modelo XL da Geonics Ltda, cedidos pelo laboratório de Geofísica aplicada da Universidade Federal do Pampa.

O resistivímetro Syscal Pro combina um transmissor, um receptor e uma unidade de comutação num único equipamento, com tensão máxima de $800 \mathrm{~V}$, corrente máxima de 2,5 A, resolução de $1 \mu \mathrm{V}$ e dez canais de leitura.

$\mathrm{O}$ equipamento foi programado com as seguintes configurações: $800 \mathrm{~V}$ de tensão transmitida, tempo de leitura de $2000 \mathrm{~ms}$ em janela única com $160 \mathrm{~ms}$ de abertura. A intensidade de corrente transmitida durante a aquisição de dados variou entre 2,1 A e 2,3 A. Foi adotado como controle de qualidade durante a aquisição de dados, leituras de medidas com desvio padrão abaixo de $5 \%$.

Foram utilizados eletrodos cerâmicos não polarizáveis preenchidos por solução de sulfato de cobre $\left(\mathrm{CuSO}_{4}\right)$ supersaturada, caracterizados por uma haste de cobre em seu interior e base porosa, que permite o trânsito da solução e percolação no solo.

Este procedimento reduz a resistência de contato e minimiza a geração de correntes parasitárias geradas pelo uso de eletrodos metálicos.

O equipamento EM-34 permite a leitura direta da condutividade elétrica em subsuperfície a partir da técnica de baixo número de induções. $\mathrm{O}$ princípio de funcionamento do equipamento decorre da passagem de corrente elétrica alternada por meio de uma bobina transmissora, responsável pela geração de um campo eletromagnético primário, que ao propagar-se em um meio condutor no subsolo, gera correntes secundárias alternadas que produzem um campo eletromagnético secundário.

Uma bobina receptora posicionada dentro da área de influência dos campos eletromagnéticos primário e secundário capta a corrente induzida resultante da soma dos campos gerados. As diferenças de intensidade, direção e fase entre os campos eletromagnético primário e secundário podem revelar a presença de corpos condutores (McNeill, 1980a).
O intervalo de condutividade registrada varia de 0 a $1000 \mathrm{mS} / \mathrm{m}$. O nível de ruído do equipamento é de $0,2 \mathrm{mS} / \mathrm{m}$. A precisão da medida é de +- $2 \%$ da deflexão da escala completa.

As frequências de operação variam conforme o espaçamento entre as bobinas. Funciona a temperaturas de $-40^{\circ} \mathrm{C}$ até $+50^{\circ} \mathrm{C}$, fora destes parâmetros de temperaturas pode apresentar erros nas leituras e até mesmo nem funcionar (GEONICS, 2007).

Os métodos geofísicos da eletrorresistividade e do eletromagnético no domínio da frequência foram aplicados ao longo de quatro perfis, com $480 \mathrm{~m}$ de comprimento cada, paralelos entre si e separados a cada $90 \mathrm{~m}$, orientados segundo a direção N125 (Figura 4).

O centro dos perfis foi planejado para que coincidisse com a posição da ocorrência mineral reconhecida em campo.

O método da eletrorresistividade foi desenvolvido a partir da técnica de caminhamento elétrico com arranjo entre eletrodos do tipo Wenner-Schlumberger. O espaçamento entre eletrodos foi de $20 \mathrm{~m}$ $(\mathrm{AB}=\mathrm{MN}=40 \mathrm{~m})$.

As leituras de resistividade elétrica resultantes foram adquiridas em 10 níveis de investigação em profundidade. A profundidade máxima de investigação decorrente do espaçamento entre eletrodos e comprimento do perfil foi de aproximadamente $90 \mathrm{~m}$.

O método eletromagnético no domínio da frequência também foi aplicado a partir da técnica de caminhamento. As medidas de condutividade aparente foram tomadas com espaçamento médio de $20 \mathrm{~m}$ entre os pontos de leitura.

As bobinas foram operadas na frequência de $0,4 \mathrm{kHz}$ em configurações coplanares horizontais e verticais com cabo de referência de $40 \mathrm{~m}$, permitindo investigações para até 60 $\mathrm{m}$ de profundidade.

Detalhes quanto aos princípios teóricos dos métodos, propriedades físicas dos materiais geológicos, assim como os principais fenômenos físicos envolvidos são descritos em Telford et al. (1976), Kearey et al. (2002), Milsom (2003) e Lowrie (2007). 
Os dados adquiridos no caminhamento elétrico foram processados no software Res2Dinv, desenvolvido pela Geotomo Software. O Res2dinv é um programa que determina, por inversão, um modelo de profundidade bidimensional para a subsuperfície a partir de dados de resistividade e cargabilidade aparentes (Griffiths \& Barker, 1993).

As medidas são transformadas por inversão em blocos de isovalores para cada nível de profundidade investigado, cujo contato entre camadas de blocos é relacionado aproximadamente à profundidade equivalente de investigação dos pontos dispostos ao longo de uma linha, com espaçamento constante entre eletrodos (Edwards, 1977). A subrotina de modelagem direta é usada para cálculo dos valores, além da técnica de otimização não linear de mínimos quadrados para a rotina de inversão (Degroot-Hedlin \& Constable 1990; Loke \& Barker, 1996).

Os dados adquiridos no caminhamento eletromagnético foram processados no software Surfer, desenvolvido pela Golden Software Inc. O Surfer é um pacote de programas gráficos que pode ser utilizado para o cálculo e a confecção de seções e mapas de variáveis a partir da interpolação de dados regularmente distribuídos.

O algoritmo de interpolação utilizado foi o de mínima curvatura, segundo o qual são efetuados cálculos de derivação repetidamente até que seja alcançada uma convergência entre os valores amostrados e os valores estimados.

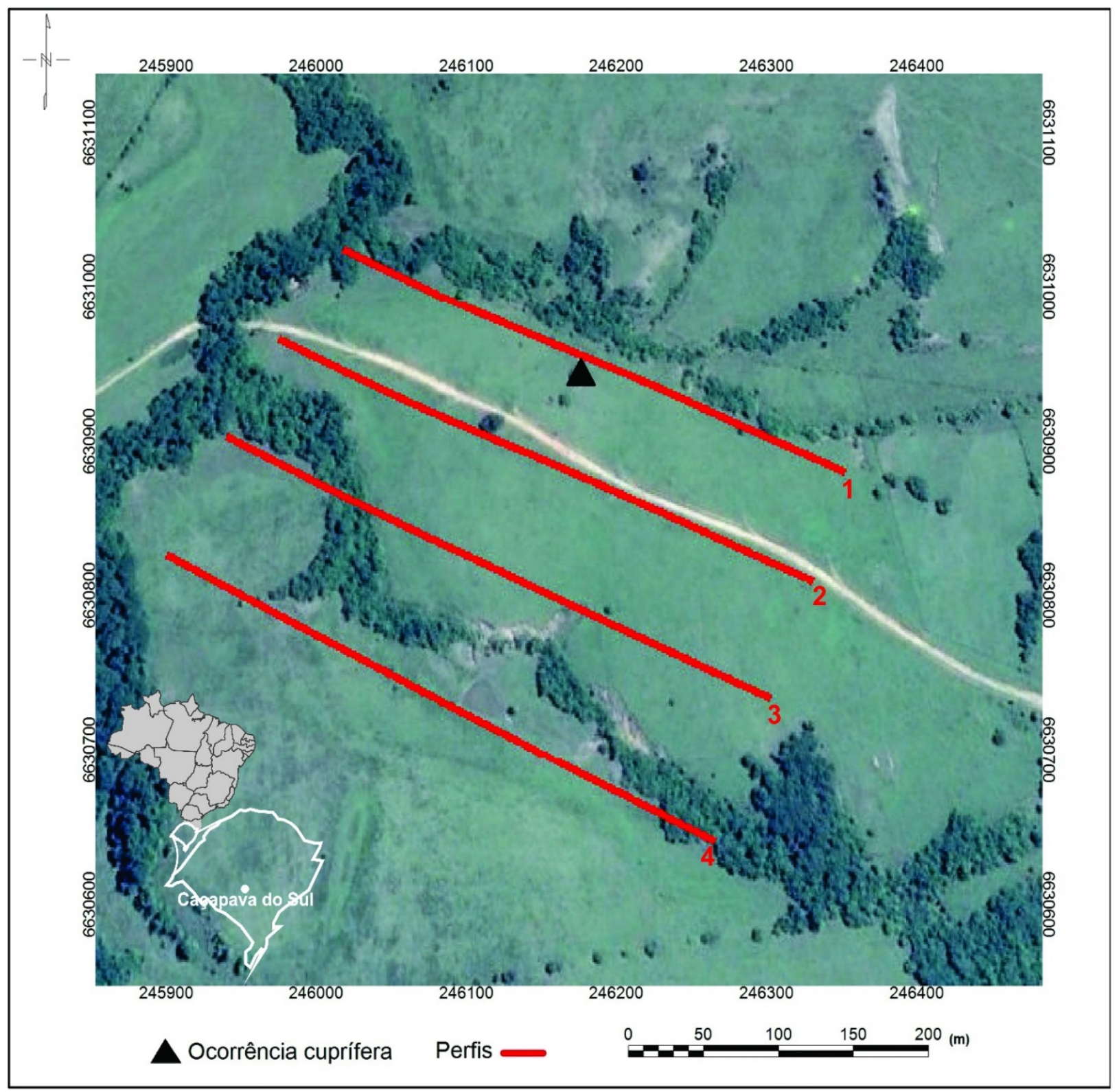

Figura 4 - Croqui de localização dos perfis de caminhamento elétrico e eletromagnético com a localização da ocorrência de cobre. 


\section{RESULTADOS E DISCUSSÕES}

Os dados geofísicos processados são apresentados em termos de modelos de inversão da resistividade e em termos de seções de condutividade aparente (Figuras 5, 6, 7 e 8). Os modelos de inversão e as seções tiveram suas respostas padronizadas em escala única de cores e valores, para que sejam permitidas análises comparativas entre os perfis. Cores frias representam valores baixos, enquanto que cores quentes caracterizam os valores mais altos.

Estudos geofísicos baseados em dados adquiridos na superfície terrestre visam essencialmente à determinação de contrastes de parâmetros físicos intrínsecos ao ambiente geológico. A ocorrência mineral estudada compreende impregnações de carbonatos de cobre em fraturas e porções mais porosas de arenitos arcosianos, que estão assentados sobre um embasamento xistoso. Esse contexto indica um claro contraste de propriedades físicas, onde minerais associados aos carbonatos e possíveis sulfetos de cobre apresentam maior condutividade em relação aos minerais constituintes dos arenitos arcosianos, e o embasamento xistoso se diferenciaria de ambos pela alta resistividade.

O trabalho de Moreira \& Ilha (2011) reconheceu áreas aproximadamente circulares de baixa resistividade em profundidades maiores que $45 \mathrm{~m}$, localizadas muito próximas à exposição da ocorrência cuprífera Capão Grande. A baixa resistividade atribuída a essas áreas foi correlacionada às mineralizações em subsuperfície, devido ao comportamento condutivo do cobre em contraste com o comportamento resistivo do arenito arcosiano conglomerático.

O modelo de inversão do perfil 1 é caracterizado pelo aumento sucessivo dos valores da resistividade com o incremento da profundidade, sob a forma de camadas paralelas que parecem acompanhar a topografia (Figura 5 - painel superior). Os valores variam de 20.000 $\Omega \mathrm{m}$, próximo a superfície, até valores superiores a $600.000 \Omega \mathrm{m}$ na base. Apenas no início da seção que é caracterizado um pequeno intervalo com baixos valores de resistividade, variando entre 7. $000 \Omega \mathrm{m}$ e $10.000 \Omega \mathrm{m}$, definido como Área 1. Essa área apresenta pouca extensão lateral e em profundidade não ultrapassa os $20 \mathrm{~m}$ (Figura 5 - painel superior).

A seção de condutividade aparente apresenta uma predominância de valores medianos a baixos $(8 \mathrm{mS} / \mathrm{m}$ a $14 \mathrm{mS} / \mathrm{m})$ para a porção inicial até a central e valores medianos a altos (15 mS/m a $20 \mathrm{mS} / \mathrm{m}$ ) a partir da projeção da estação $280 \mathrm{~m}$ até o final do perfil (Figura 5 painel inferior). A área 1 delimitada no modelo de inversão de resistividade se encontra na faixa de valores médios a baixos de condutividade aparente (Figura 5 - painel inferior).O intervalo de baixa resistividade da Área 1 é interpretado como uma porção espessa de solo, provavelmente saturado em água. Intervalos de valores de resistividade variando entre $16.000 \Omega \mathrm{m}$ e $636.000 \Omega \mathrm{m}$ são associados as camadas dos arenitos arcosianos da Formação Passo da Promessa. Valores de resistividade mais elevados, correspondentes a profundidades superiores a $70 \mathrm{~m}$, são definidos como a resposta dos xistos do embasamento cristalino (Complexo Metamórfico Vacacaí).

O modelo de inversão da resistividade do perfil 2 apresenta uma mudança significativa no padrão estratificado das camadas em subsuperfície, observado na figura 5, principalmente na sua porção central, próxima a projeção da ocorrência mineral, onde um intervalo de baixos valores de resistividade continuam até $50 \mathrm{~m}$ de profundidade, denominado Área 3 (Figura 6 - painel superior). Essa área está envolvida por valores intermediários de resistividade, que variam entre $25.000 \Omega \mathrm{m}$ e $100.000 \Omega \mathrm{m}$.

Também foram caracterizadas áreas horizontais de baixa resistividade (Área 1, 2 e 4), cujas profundidades giram em torno de 20 m. A base da seção é demarcada por camadas dobradas de elevada resistividade (Figura 6 painel superior).

A seção de condutividade aparente (Figura 6 - painel inferior) mostra que os intervalos correspondentes às Áreas 1, 2 e 4 demarcadas no modelo de resistividade estão caracterizadas por valores médios a altos de condutividade aparente $(15 \mathrm{mS} / \mathrm{m}$ a $19 \mathrm{mS} / \mathrm{m})$. O intervalo representativo da Área 3 corresponde a valores intermediários de condutividade aparente (12 $\mathrm{mS} / \mathrm{m})$. 


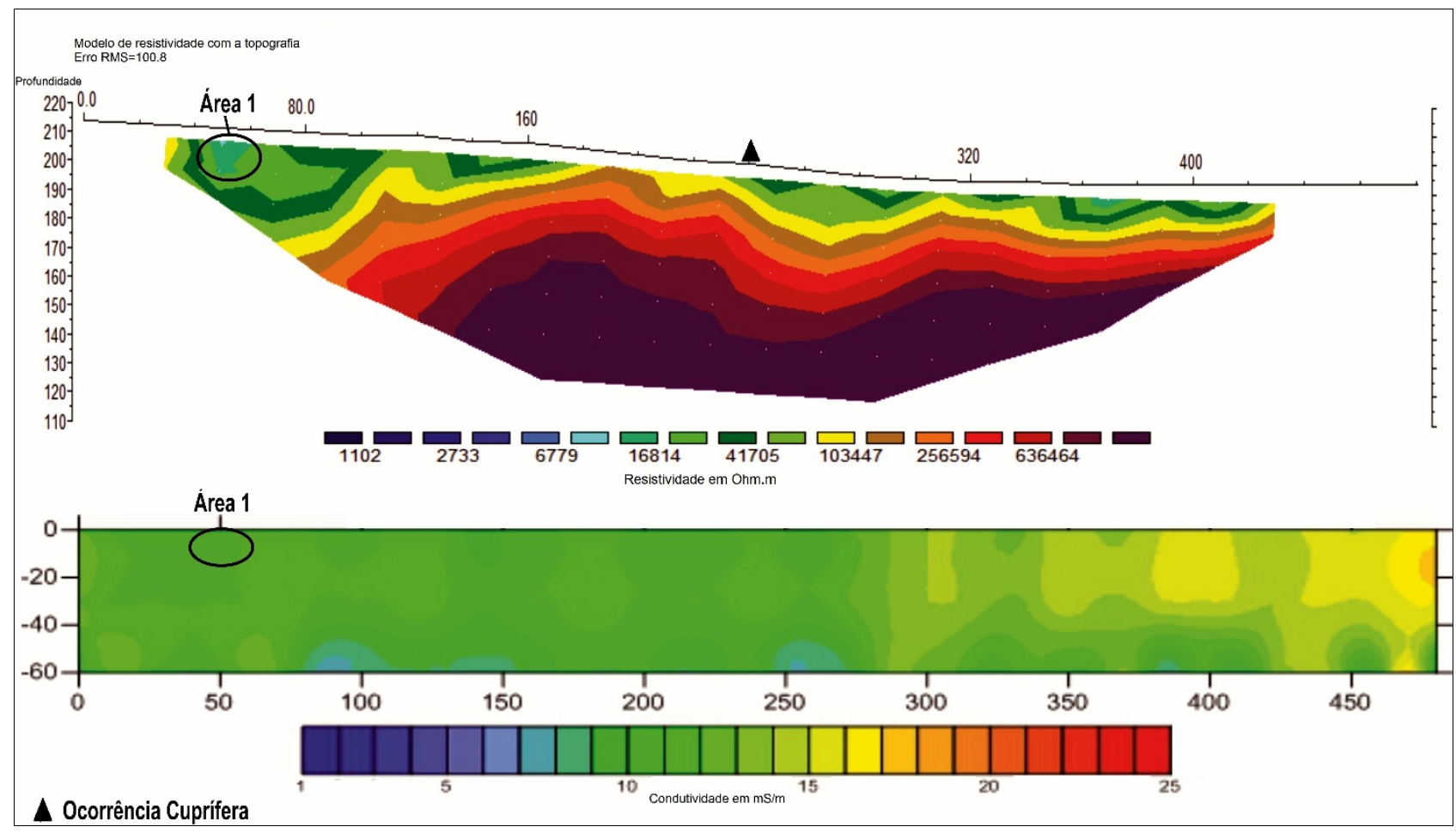

Figura 5 - Resultados dos caminhamentos elétrico e eletromagnético do perfil 1: Modelo de inversão de resistividade (painel superior); Seção de condutividade aparente (painel inferior).

Os intervalos compreendidos pelas Áreas horizontais rasas 1 , 2 e 4 são explicados como uma porção do solo saturado em água, em semelhança a Área 1 do modelo de inversão anterior (vide Figura 5). O intervalo de baixa resistividade da Área 3 foi associado a continuidade da ocorrência mineral em subsuperfície, devido a verticalidade da área e ao seu posicionamento nas proximidades da projeção do afloramento mineralizado. Os valores de condutividade intermediários caracterizados para essa área sugerem apenas a ocorrência de carbonatos de cobre em profundidade. Os valores resistivos na base são associados às rochas cristalinas do embasamento.

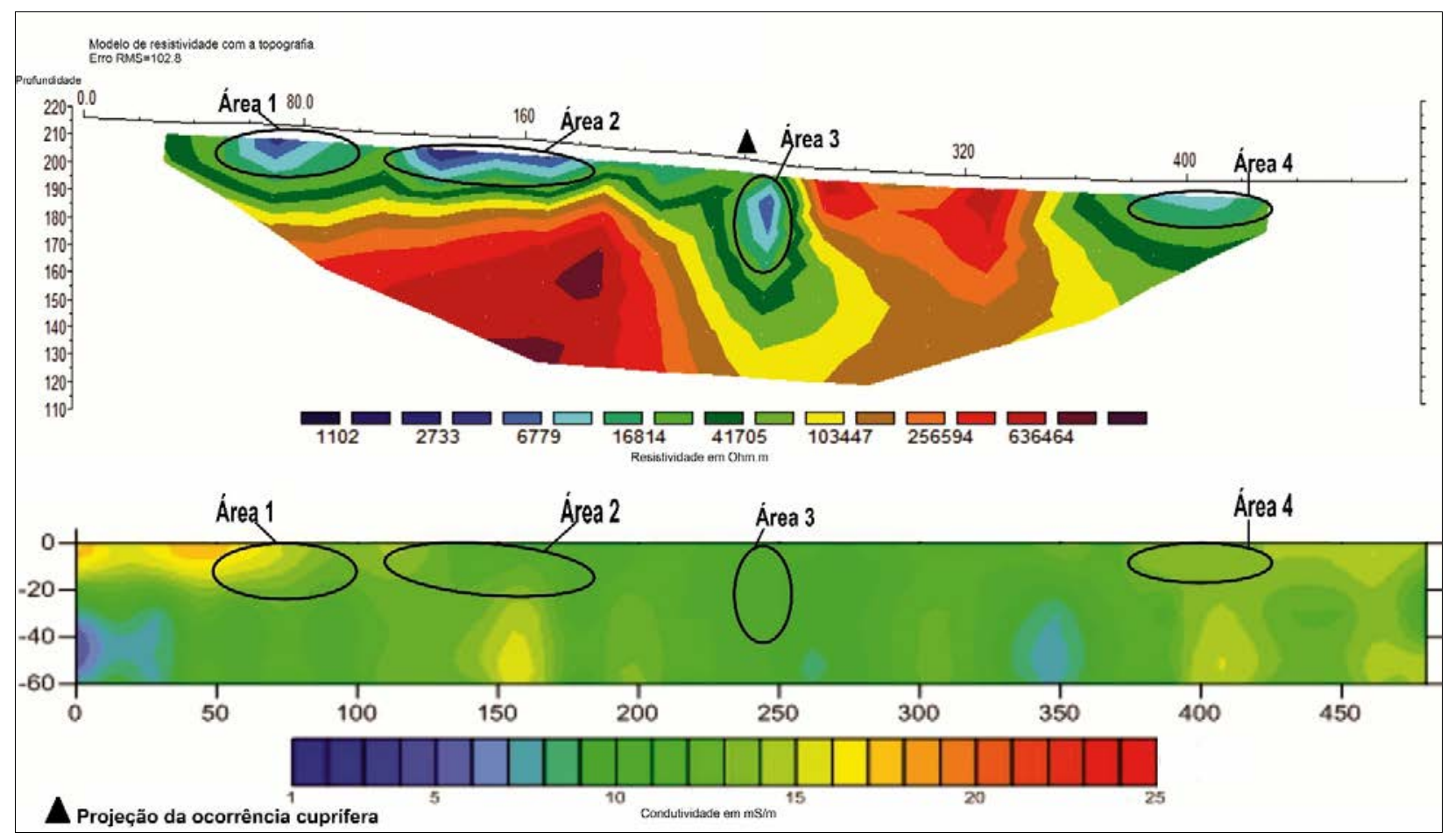

Figura 6 - Resultados dos caminhamentos elétrico e eletromagnético do perfil 2: Modelo de inversão de resistividade (painel superior); Seção de condutividade aparente (painel inferior). 
O modelo de inversão do perfil 3 evidencia um predomínio de áreas de baixa resistividade ocupando tanto porções mais próximas a superfície quanto da base (Figura 7 -painel superior).

As duas primeiras áreas (1 e 2) ocupam praticamente os primeiros $200 \mathrm{~m}$ do perfil. Apresentam valores de resistividade variando entre $4.000 \Omega \mathrm{m}$ e $11.000 \Omega \mathrm{m}$ para até $25 \mathrm{~m}$ de profundidade.

A Área 3 está situada na porção central do perfil, coincidente com a posição da projeção da exposição da ocorrência mineral. Apresenta valores de resistividade em torno de $9.000 \Omega \mathrm{m}$, forma circular e atinge profundidades da ordem de $50 \mathrm{~m}$. A área 4 está compreendida entre as estações de $290 \mathrm{~m}$ e $320 \mathrm{~m}$.

Apresenta valores de resistividade ainda mais baixos $(4.000 \Omega \mathrm{m})$ e alcançam os $70 \mathrm{~m}$ de profundidade. A área 5 se destaca por ser a mais profunda ( $80 \mathrm{~m})$ e a mais condutiva $(2.000$ $\Omega \mathrm{m})$.

Valores altos de resistividade (400.000 $\Omega$ $\mathrm{m})$, na forma de camadas paralelas que mergulham para SW dominam a porção basal do modelo (Figura 7 - painel superior).

A seção de condutividade aparente apresenta valores medianos a altos (15 mS/m a $20 \mathrm{mS} / \mathrm{m})$ ladeando índices baixos a médios $(9 \mathrm{mS} / \mathrm{m}$ a 13 $\mathrm{mS} / \mathrm{m}$ ) entre as estações de $260 \mathrm{~m}$ e $390 \mathrm{~m}$ (Figura 7 - painel inferior).

As Áreas 1 e 2 demarcadas no modelo de inversão de resistividade estão situadas em porções com os valores condutivos mais elevados da seção (atingem $20 \mathrm{mS} / \mathrm{m}$ ).

A Área 3 está localizada no limite da zona de valores condutivos medianos a altos. As Áreas 4 e 5 praticamente estão localizadas no intervalo de valores condutivos medianos a baixos, com exceção da extremidade sudeste da Área 5, onde a condutividade aumenta (Figura 7 -painel inferior).

Os intervalos de baixa resistividade das Áreas 1 e 2 são interpretados como porções do solo saturado em água. O intervalo central da Área 3 foi novamente associado a continuação em subsuperfície dos carbonatos de cobre encontrados no afloramento mineralizado. Nessa área também podem estar presentes sulfetos devido os valores médios a altos de condutividade aparente.

Os intervalos das Área 4 e 5 também são explicados como possíveis acumulações somente de carbonatos de cobre em subsuperfície, visto os valores medianos a baixos de condutividade aparente.

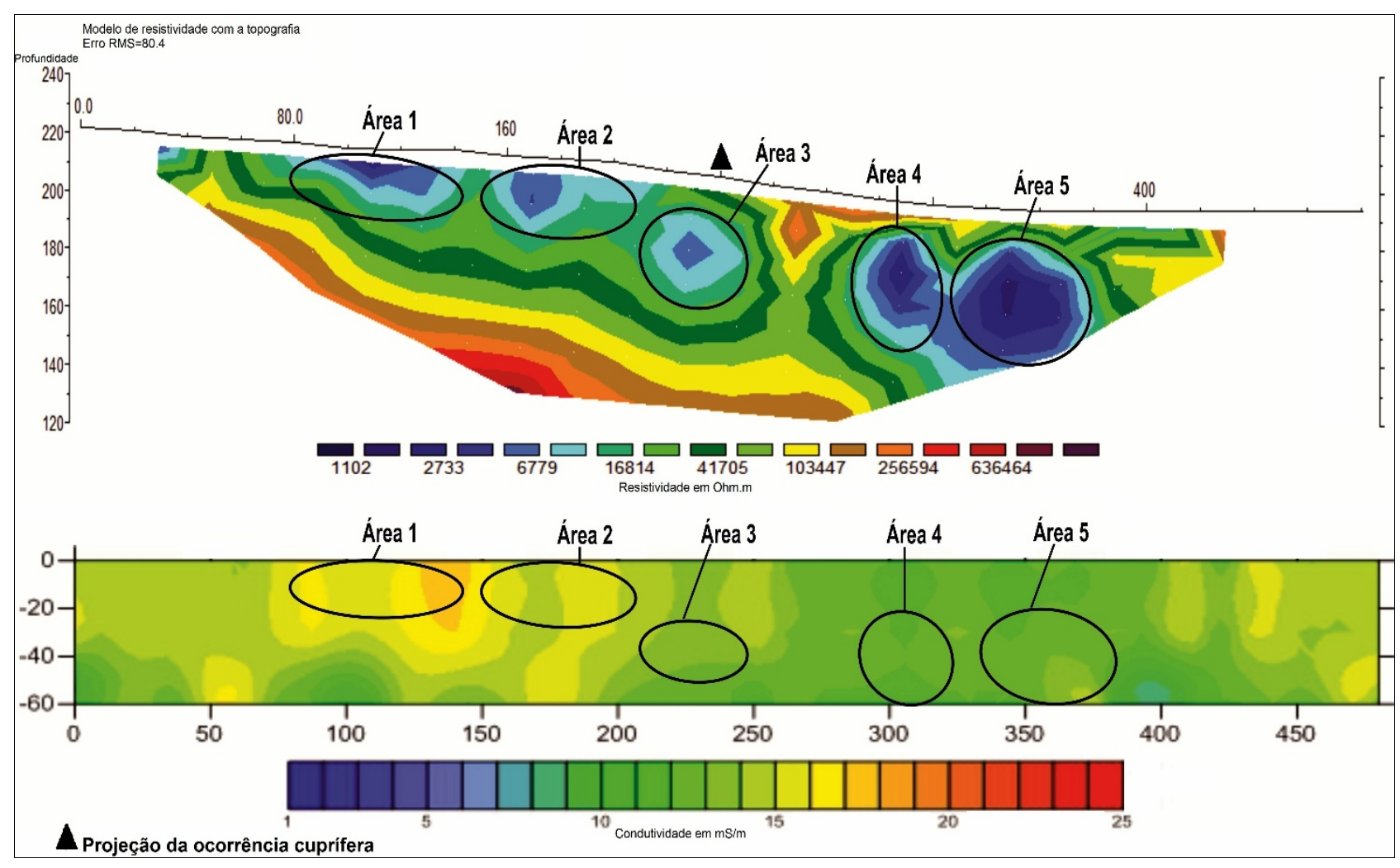

Figura 7 - Resultados dos caminhamentos elétrico e eletromagnético do perfil 3: Modelo de inversão de resistividade (painel superior); Seção de condutividade aparente (painel inferior). 
O modelo de inversão do perfil 4 mostra três áreas com valores baixos de resistividade (Figura 8 -painel superior). $\mathrm{O}$ intervalo compreendido pela Área 1 é o que apresenta os valores mais baixos de resistividade (3.000 $\Omega$ $\mathrm{m})$ e o que alcança a maior profundidade (65m).

O intervalo de baixos valores de resistividade da Área 2 tem início na estação de 240 m (posição da projeção do afloramento mineralizado) e fim na estação de $280 \mathrm{~m}$. Apresenta forma achatada e alcança os $50 \mathrm{~m}$ de profundidade.

O intervalo da Área 3 é o mais extenso e o mais próximo à superfície (não ultrapassa os 25 m). A base é novamente demarcada por camadas horizontais inclinadas de elevada resistividade.

A seção de condutividade aparente é caracterizada por valores intermediários (em torno de $12 \mathrm{mS} / \mathrm{m}$ ) rodeando valores médios a altos entre as estações de $70 \mathrm{~m}$ e $370 \mathrm{~m}$ (Figura 8 - painel inferior). A área 1 demarcada no modelo de inversão de resistividade é a mais condutiva da seção (alcança $22 \mathrm{mS} / \mathrm{m}$ ). O intervalo da Área 2 está inserida na porção de valores de condutividade médios a altos. A Área 3 está inserida na faixa de transição de valores médios a altos para médios a baixos de condutividade aparente.

As Áreas 2 e 3 só foram assim delimitadas por meio da observação da variação da condutividade conforme a profundidade. O modelo de inversão da resistividade apontava para uma grande área.

O intervalo de baixa resistividade da Área 1 é interpretado como uma provável acumulação de sulfetos em subsuperfície, devido a correlação entre a baixa resistividade e a alta condutividade.

A Área 2 foi associada a possível continuidade em profundidade dos carbonatos de cobre, identificados no afloramento mineralizado, com eventuais sulfetos subordinados, assim explicados com base nos valores médios a altos de condutividade aparente. A Área 3, próxima a superfície, é considerada como uma porção saturada de solo.

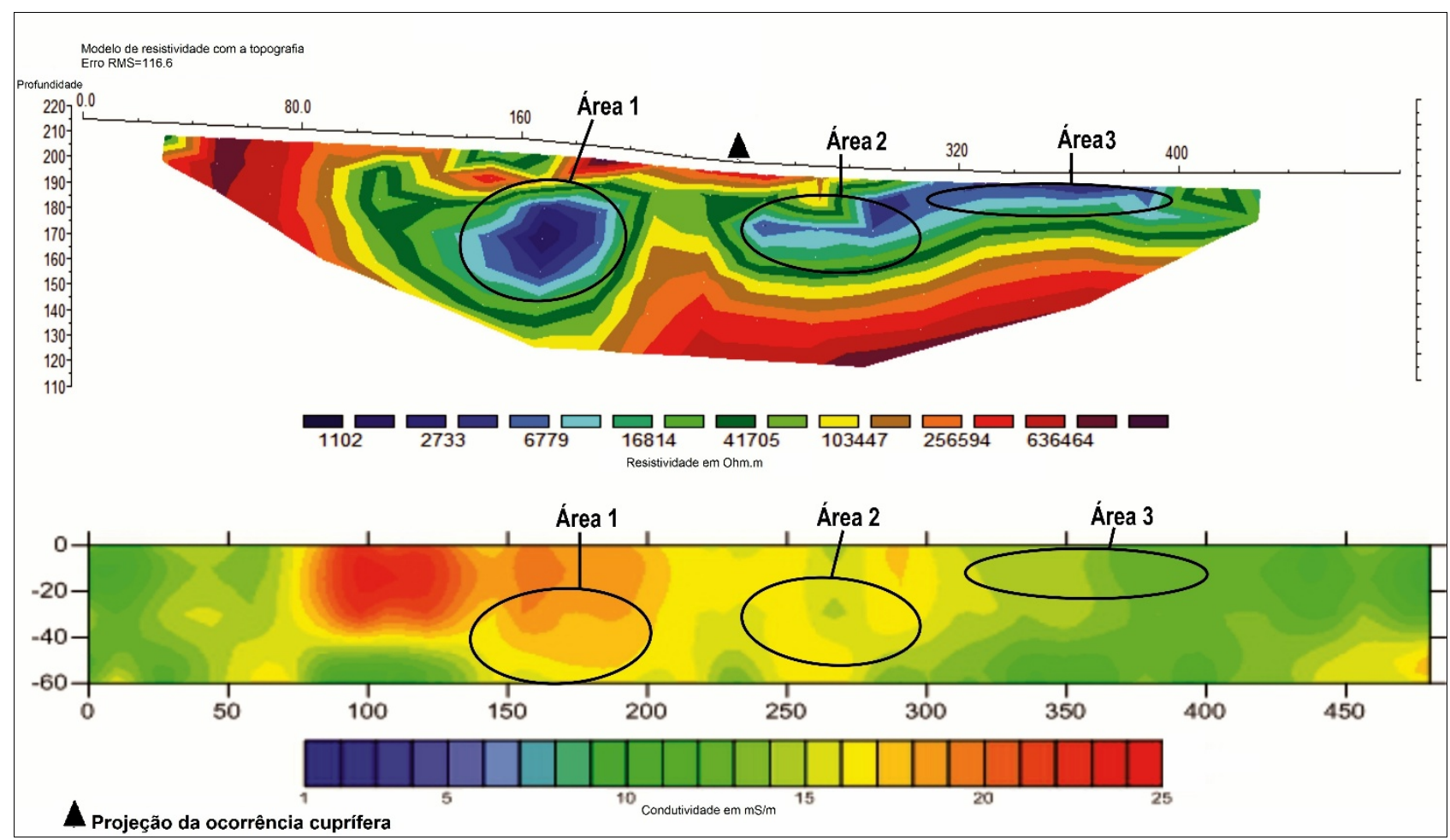

Figura 8 - Resultados dos caminhamentos elétrico e eletromagnético do perfil 4: Modelo de inversão de resistividade (painel superior); Seção de condutividade aparente (painel inferior).

A dispersão das áreas de baixa resistividade supostamente associadas a mineralização em subsuperfície, ao longo dos perfis 2, 3 e 4 (Figuras 6, 7 e 8) sugere sua continuidade para sudoeste do afloramento mineralizado (Figura
9). Em oposição, a ausência dessas áreas no perfil 1 mostra que a mineralização não se prolonga para norte da ocorrência.

$\mathrm{O}$ alinhamento das áreas de baixa resistividade revela a mesma orientação $\mathrm{N} 20^{\circ}$ 
das principais mineralizações de cobre no âmbito da Bacia do Camaquã (Figura 9).

As direções $\mathrm{N}_{22^{\circ}}$, N56 ${ }^{\circ}$ e $\mathrm{N}^{\circ} 28^{\circ}$ representam as principais famílias de fraturas mineralizadas descritas na ocorrência mineral, um indicador do controle estrutural da mineralização. Contudo, é possível que o sistema de falhas tenha servido de conduto para ascensão de fluídos hidrotermais que posteriormente percolaram zonas de maior porosidade no arenito, possivelmente associada a feições ou fácies sedimentares originalmente formadas durante a sedimentação da unidade. Diante das evidências, é provável que exista um controle estrutural e litológico na orientação da zona mineralizada.

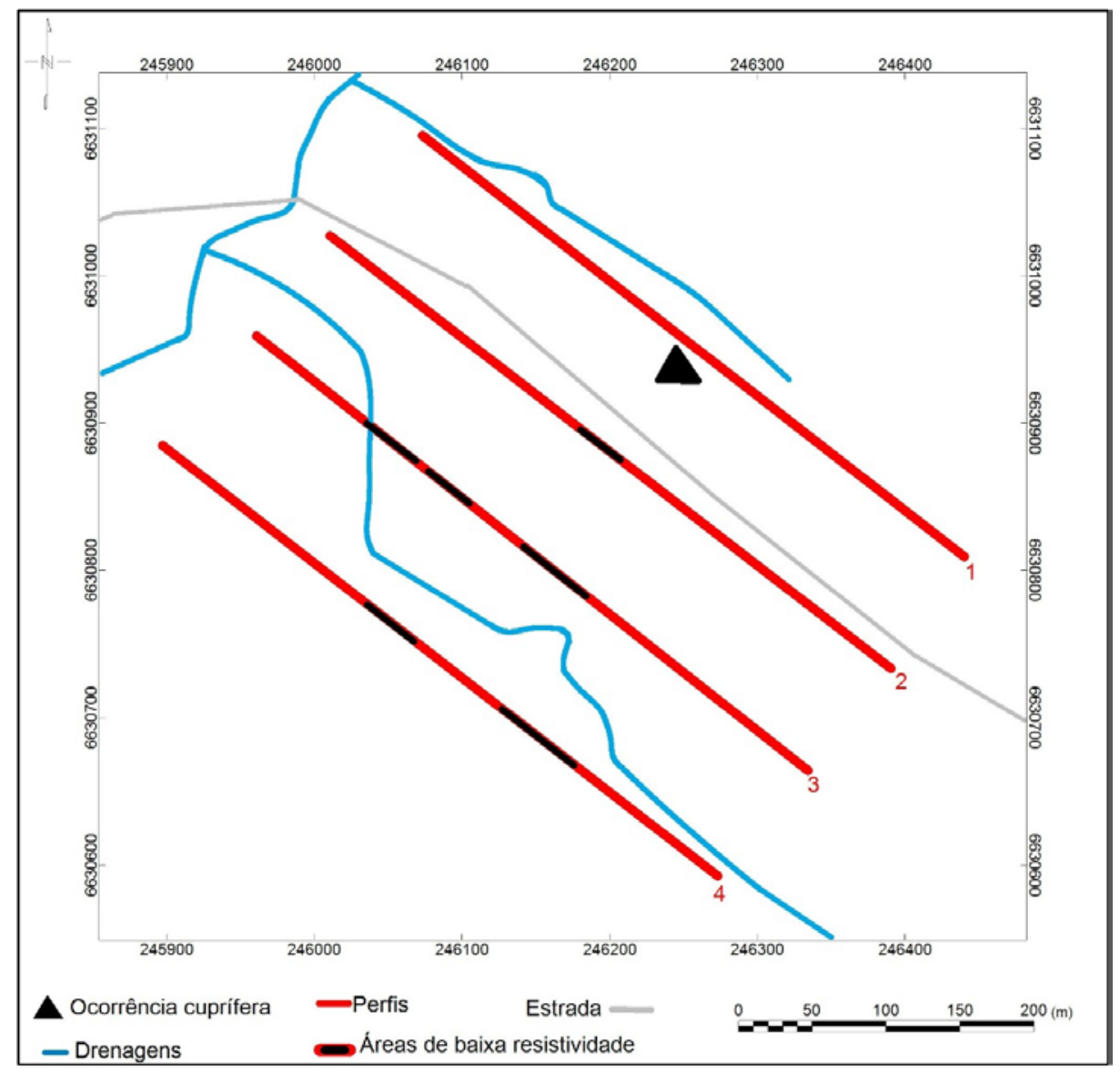

Figura 9 - Dispersão das áreas condutivas associadas a mineralização na área de estudo.

\section{CONCLUSÕES}

O parâmetro resistividade elétrica foi bastante sensível na obtenção de contrastes de propriedades físicas entre a rocha encaixante e a mineralização da ocorrência cuprífera em subsuperfície.

Elevados valores de resistividade foram atribuídos as rochas do embasamento cristalino, índices moderados a altos foram sugestivos de arenitos arcosianos encaixantes e valores baixos foram relacionados a possíveis mineralizações de cobre.

As áreas de baixa resistividade supostamente associadas a mineralização foram caracterizadas com formas aproximadamente circulares para profundidades de até $80 \mathrm{~m}$. Já as áreas equivalentes horizontalizadas, normalmente superficiais (até $25 \mathrm{~m}$ de profundidade), foram relacionadas a solos espessos provavelmente saturados em água

O alinhamento da disposição preferencial das áreas de baixa resistividade atribuídas a mineralização ao longo da área de estudo mostrou a mesma orientação N20 ${ }^{\circ}$ atribuída por Teixeira (1937), Leinz \& Almeida (1941) e Costa Filho (1944) como a preferencial para as mineralizações cupríferas clássicas da Bacia Sedimentar do Camaquã.

A família de fraturas mineralizadas $\mathrm{N} 18^{\circ}$ encontrada no afloramento Capão Grande é muito próxima a orientação preferencial N20 ${ }^{\circ}$ das áreas atribuídas a mineralização. Essa evidencia sugere o controle estrutural da mineralização. 
O parâmetro condutividade elétrica teve baixa sensibilidade na identificação de contrastes de propriedades físicas ente a ocorrência mineral e a rocha encaixante. Somente com os dados de caminhamento eletromagnético não seria possível a identificação da mineralização em subsuperfície.

Os dados de condutividade aparente tiveram utilidade em análises comparativas com as áreas prévias de baixa resistividade associadas a mineralização pelos dados de caminhamento elétrico. Uma análise possibilitou a delimitação de áreas distintas, primariamente apontadas pelos dados de resistividade como uma grande área. Demais correlações esperadas de alta condutividade/baixa resistividade possibilitaram estimativas da presença de sulfetos em subsuperfície.

A distância entre pontos de leitura diante da dimensão restrita das áreas de baixa resistividade pode ser o fator que colaborou para a diferença de sensibilidade na definição de áreas associadas a mineralização em subsuperfície pelos dados de caminhamento eletromagnético.

Os dados de caminhamento elétrico forneceram os subsídios necessários para a definição da continuidade lateral e em profundidade da zona com provável mineralização para sudoeste da ocorrência cuprífera Capão Grande.

Os dados de caminhamento eletromagnético permitiram estimativas da presença de sulfetos em subsuperfície.

\section{AGRADECIMENTOS}

Os autores agradecem ao Laboratório de Geofísica Aplicada da Universidade Federal do Pampa, câmpus Caçapava do Sul, pela concessão dos equipamentos utilizados em campo, assim como aos proprietários da fazenda Dona Rosa pela liberação do acesso à área de estudo.

\section{REFERÊNCIAS}

ALLIS, R. G. Geophysical anomalies over epithermal systems. Journal of Geochemical Exploration, v. 36, n. 1, p. 339374, 1990.

BOCCHI, P. R. Geologia da Folha de Caçapava do Sul, 1970. Rio Grande do Sul. Boletim da Divisão de Geologia e Mineralogia. DNPM, Rio de Janeiro: $\mathrm{n}^{\circ}$ 245, P 1-83.

COSTA FILHO, J. H. Prospeç̧ão das minas de cobre de Camaquã, Rio Grande do Sul. Departamento Nacional de Produção Mineral - DNPM. Ministério da Agricultura. Boletim. N. 59. p. 13 - 94. 1944.

CHATTERJEE, K. K. Uses of Metals and metallics minerals. New Age International, 333p., 2007.

CPRM - COMPANHIA DE PESQUISA DE RECURSOS MINERAIS - SERVIÇO GEOLÓGICO DO BRASIL. Mapa Geológico do Rio Grande do Sul. Programa de Levantamentos Geológicos Básicos do Brasil. Escala 1:750000. Porto Alegre, 2007.

DEGROOT-HEDLIN, C. \& CONSTABLE, S. Occam's inversion to generate smooth two-dimensional models form magnetotelluric data. Geophysics, v. 55, p. 1613- 1624, 1990.

DOYLE, H. Geophysical exploration for gold - a review. Geophysics, v. 55, n. 2, p. 134-146, 1990.

EDWARDS, L.S. A modified pseudosection for resistivity and induced-polarization. Geophysics, v. 42, p. 1020-1036, 1977.

FALLON, T. \& BACKO, J. Archaean BIF-hosted gold, Mount Morgans, Western Australia: a geophysical case history. Exploration Geophysics, v. 25, n. 3, p. 166-166, 1994.

GARRIDO, I. A. A. Estudos Geofísicos Detalhados da Área Sítio do Chico. Salvador, 1978. 86p. (Dissertação de Mestrado), Universidade Federal da Bahia.

GEONICS. EM-34 \& EM34-3XL Operating Instructions (for model with two digital meters). Ontario Canada L5T 1C6, 2007.

GRIFFITHS, D. H. \& BARKER, R. Two-dimensional resistivity imaging and modeling in areas of complex geology. Journal of Applied Geophysics, v. 29, p. 211-226, 1993.

IRVINE, R. J. \& SMITH, M. J. Geophysical exploration for Epitermal deposits. Journal of Geochemical Exploration, v.
36, p. 375 - 412, 1990.

JACKSON, J. The use of sub-audio magnetics (SAM) in gold exploration - examples from the Yilgarn Craton, WA. Exploration Geophysics, v. 36, n. 2, p.163-169, 2005.

KEAREY, P.; BROOKS, M.; HILL, I. An Introduction to Geophysical Exploration. 3. Ed. Oxford. Blackwell: Blackwell Science Ltd. 2002.

LAUX, J. H. \& LINDENMAYER, Z. G. As Minas do Camaquã: um século de evolução de hipóteses genéticas. In: RONCHI, L.H.; LOBATO, A.O.C. (Eds). Minas do Camaquã: um estudo multidisciplinar. São Leopoldo: Editora Unisinos, 2000, p. 133-164.

LAUX, J. H.; LINDENMAYER, Z. G.; TEIXEIRA, J. B.; BASTOS NETO, A. Ore genesis at the Camaquã copper mine, a neoproterozoic sediment-hosted deposit in Southern Brazil. Ore Geology Reviews, v. 26, p. 71-89, 2005.

LEINZ, V. \& ALMEIDA, S. C. Gênese da jazida de cobre do Camaquã. Porto Alegre, Secretaria de Estado dos Negócios da Agricultura, Indústria e Comércio, 1941.

LOKE, M.H. \& BARKER, R. Rapid least-squares inversion of apparent resistivity pseudosections by a quasi-Newton method. Geophysical Prospecting, v. 44, p. 131-152, 1996.

LOWRIE, W. Fundamentals of Geophysics, sec. edit. Cambridge University Press, p 207-219, 2007

MAAS M. V. R., OLIVEIRA C. G., PIRES A. C. B., MORAES R. A. V. Aplicação da geofísica aérea na exploração mineral e mapeamento geológico do setor sudoeste do Cinturão Cuprífero Orós-Jaguaribe. Revista Brasileira de Geociências, v. 33, n. 3, p. 279-288 2003.

MILSOM, J. Field Geophysics. Chichester, John Wiley \& Sons, 249p., 2003.

MOON, C.J.; Whateley, M.E.G. \& Evans, A.M. Introduction to Mineral Exploration. 2. ed. Oxford: Blackwell Publishing, Malden, USA, 499p. 2006.

MOREIRA, C. A. \& ILHA, L. M. Prospecção Geofísica em ocorrência de cobre localizada na bacia sedimentar do Camaquã (RS). Revista da Escola de Minas, v. 64, n. 3, p. 309- 315, 2011. 
MOREIRA, C. A., LOPES, S. M., SCHWEIG, C.; SEIXAS, A. R. Geoelectrical Prospection of disseminated Sulfide Mineral occurrences in Camaquã Sedimentary Basin, Rio Grande do Sul State, Brazil. Revista da Sociedade Brasileira de Geofísica, v. 30, n. 2, p. 169-179, 2012

McNEILL, J. Electromagnetic Terrain Conductivity Measurement at Low Induction Numbers. Technical Note TN, v. 6, p. 6-15, 1980. Disp. em: <http://www.geonics .com>. Acesso em: 11 dezembro 2015.

PEREIRA, R. M. Fundamentos de Prospecção Mineral. Rio de Janeiro. Editora: Interciência Ltda, 2003.

PORCHER, C. A.; LEITES, S. R.; RAMGRAB, G. E.; CAMOZZATO, E. Folha Passo do Salsinho.SH. 22 - Y - A - I - 4. Estado do Rio Grande do Sul. CPRM, Programa Levantamentos Geológicos Básicos do Brasil, escala 1:50.000, 372p., 1995.

RONCHI, L. H. \& LOBATO, A. O. C. Minas do Camaquã: Um Estudo Multidisciplinar. São Leopoldo. Editora: Unisinos, 366p., 2000.

SÁ, E. P. Levantamento geofísico integrado da área de Terra do sal, Curaçá-BA: Aplicação na reavaliação geológica visando à prospecção de cobre. Bahia. 1981. 98p. Dissertação (Mestrado). Universidade Federal da Bahia. (UFBA).

TELFORD, W. M.; GELDART, L.P.; SHERIFF, R.E.; KEYS, D.A. Applied Geophysics. Cambridge: Cambridge University, 1976

TEIXEIRA, E. Cobre no Rio Grande do Sul. Boletim da Divisão de Fomento da Produção Mineral, DNPM. Rio de Janeiro. Avulso 22. 29 p. 1937

WHITE, S. R. M. S.; COLLINS, R.; DENNE, R.; HEE, R.; BROWN, P. A new survey design for 3D IP inversion modelling at Copper Hill. Exploration Geophysics, v. 32, p. 152-155, 2001 